\title{
Synthesis and Characterization of Hydrogels from Graft Copolymers
}

\author{
Yasuhisa Tsukahara, Hsi-Chuan TsaI, Yuya Yamashita, \\ and Yoshio MUROGA \\ Department of Synthetic Chemistry, Faculty of Engineering, \\ Nagoya University, Nagoya 464, Japan
}

(Received January 13, 1987)

\begin{abstract}
Graft copolymers of well-defined structure composed of a 2,3-dihydroxypropyl methacrylate backbone and uniform molecular weight polystyrene branches were prepared by the macromonomer method and studied for their application as hydrogels utilizing their microdomain structure. Film specimens prepared by solution casting of the graft copolymer swelled immediately in water to form stable hydrogels which were characterized by measuring the equilibrium degree of swelling and the oxygen permeability in water. Swelling behavior of the hydrogel was also characterized by the small angle X-ray scattering (SAXS) measurement. It is clearly shown that the microphase separated structure of the graft copolymers is considerably regular and the swelling behavior and the oxygen permeability coefficient of hydrogels can be well-controlled by both the architecture of preformed graft copolymers and the microdomain structure in the cast specimens.

KEY WORDS Graft Copolymer / Macromonomer / Hydrogel /

Microphase Separated Structure / Copolymerization / Microdomain /

Swelling / Oxygen Permeability / Biomedical Application /
\end{abstract}

Hydrogels prepared from the nonionic water soluble polymers like poly(2-hydroxyethyl methacrylate), poly(propylene glycol monoacrylate), poly(vinylalcohol), and polyacrylamide have been proposed for medical applications such as surgical implants, soft contact lenses, slow release devices and some others. ${ }^{1-12}$ These polymeric materials can swell in water and contain large amount of water which is considered to be essential to their slight irritation and biocompatibility for living tissues because the interfacial free energy between water-swollen gel and the aqueous biological environment is very small and the inner water provides good permeability for oxygen, metal ions, and other metabolites. However, hydrogels at high swelling state often show insufficient mechanical strength which has been a large difficulty for their wide application.
Yamashita et al. reported on hydrogels prepared from poly(2-hydroxyethyl methacrylate (HEMA)- $g$-styrene) utilizing the microphase separated structure in the copolymer. ${ }^{13}$ They showed that the hydrogels were transparent and had much better mechanical strength compared with the crosslinked HEMA homopolymer. Furthermore, they observed pronounced thromboresistance with the hydrogel in the blood compatibility experiment. It is known at present that the microphase separated structure of the materials in biomedical applications including hydrogels acts a quite important role in improving both their mechanical strength and biocompatibility. ${ }^{14-16}$

We have been studying the synthesis and characterization of tailor-made graft copolymers having controlled graft number and length by the macromonomer method to 


\section{Y. TSUKAHARA et al.}

functionalize the polymeric materials. ${ }^{17-22}$ In this paper, we report the synthesis and characterization of hydrogels prepared from poly(2,3-dihydroxypropyl methacrylate- $g$ styrene) which were synthesized by the macromonomer method. Poly(2,3-dihydroxypropyl methacrylate) (PDHPMA) is an interesting polymer as a hydrogel because of the much higher affinity for water than poly(hydroxyethyl methacrylate) (PHEMA). ${ }^{5}$ Futhermore, it might be essential to use the well-characterized graft copolymer for understanding their biocompatibility and other properties.

\section{EXPERIMENTAL}

\section{Materials}

Isopropylideneglyceryl methacrylate (IPGMA) monomer was supplied from Toyo Contact Lens Co., Ltd. Styrene (St) monomer, glycidyl methacrylate, trifluoroacetic acid, azobisisobutyronitrile (AIBN), and other solvents are commercially available. Carbon dioxide used was supplied from Takachiho Chemical Co., Ltd. with $99.99 \%$ high purity. $s$ $\mathrm{BuLi}$ was prepared from the reaction of $s$ butylchloride with $\mathrm{Li}$ metal in $n$-hexane. AIBN was purified by recrystallization with methanol. Monomers and solvents were dried and distilled under a nitrogen atmosphere. St monomer and toluene used in the living anionic polymerization were further purified with sodium salt of benzophenone several times under high vacuum. Tetrahydrofuran (THF) was also further purified by the sodium salt of $\alpha$-methylstyrene tetramer under high vacuum.

\section{Styrene Macromonomer}

Purified toluene containing $10 \%$ THF in a glass ampoule was poured into a polymerization glass flask through a breakable seal followed by the addition of $n$-hexane solution of $s-\mathrm{BuLi}$ and toluene solution of St monomer at $-78^{\circ} \mathrm{C}$. Living anionic polymerization was carried out at $0^{\circ} \mathrm{C}$ for 10 hours under high vacuum (ca. $1 \times 10^{-7} \mathrm{mmHg}$ ). After the polymerization of styrene monomers, carbon dioxide gas was introduced into the polymerization flask through a breakable seal at $-78^{\circ} \mathrm{C}$, and the reaction mixture was stirred for 12 hours at $0^{\circ} \mathrm{C}$. The coupling reaction was confirmed by the disappearance of the yellow-red color of styryllithium anions. The coupling product was precipitated into a large amount of methanol and then freezedried with benzene. The contents of the carboxyl end group of styrene prepolymers were determined by titrations with $0.01 \mathrm{NOH}$ using TSB-10A/TSC-10A auto titrater of TDA Co., Ltd. The reaction of the terminal carboxyl group with an excess amount of glycidyl methacrylate was carried out in $\mathrm{xy}-$ lene at $140^{\circ} \mathrm{C}$ for $6-7$ hours in a glass ampoule in the presence of small amount of hydroquinone and $N, N$-dimethyllaurylamine (Scheme 1). After the reaction, the obtained St macromonomers were precipitated in methanol and freeze-dried with benzene. The reaction yield was estimated by the titration of unreacted prepolymers with $\mathrm{KOH}$. The number average molecular weight $\left(\bar{M}_{n}\right)$ and the molecular dispersity index $\left(\bar{M}_{w} / \bar{M}_{n}\right)$ were calculated from gel permeation chromatography (GPC) using the standard polystyrene (PSt) samples.

\section{Poly(2,3-dihydroxypropyl methacrylate-g-sty- rene)}

Radical copolymerization of $\mathrm{St}$ macro-
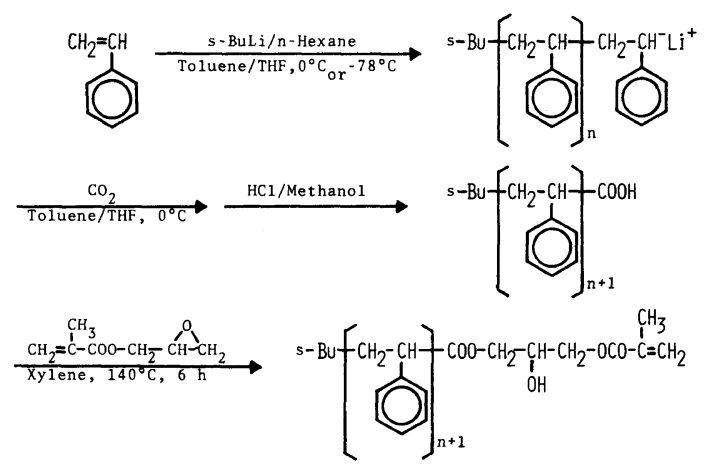

Scheme 1. Preparation of macromonomers. 


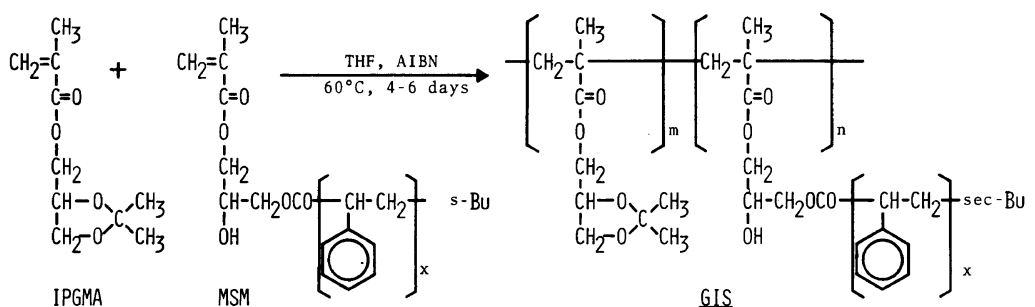

GIS

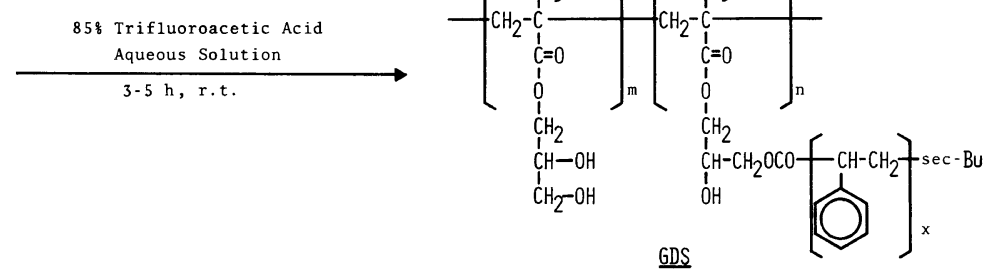

Scheme 2. Preparation of graft copolymers.
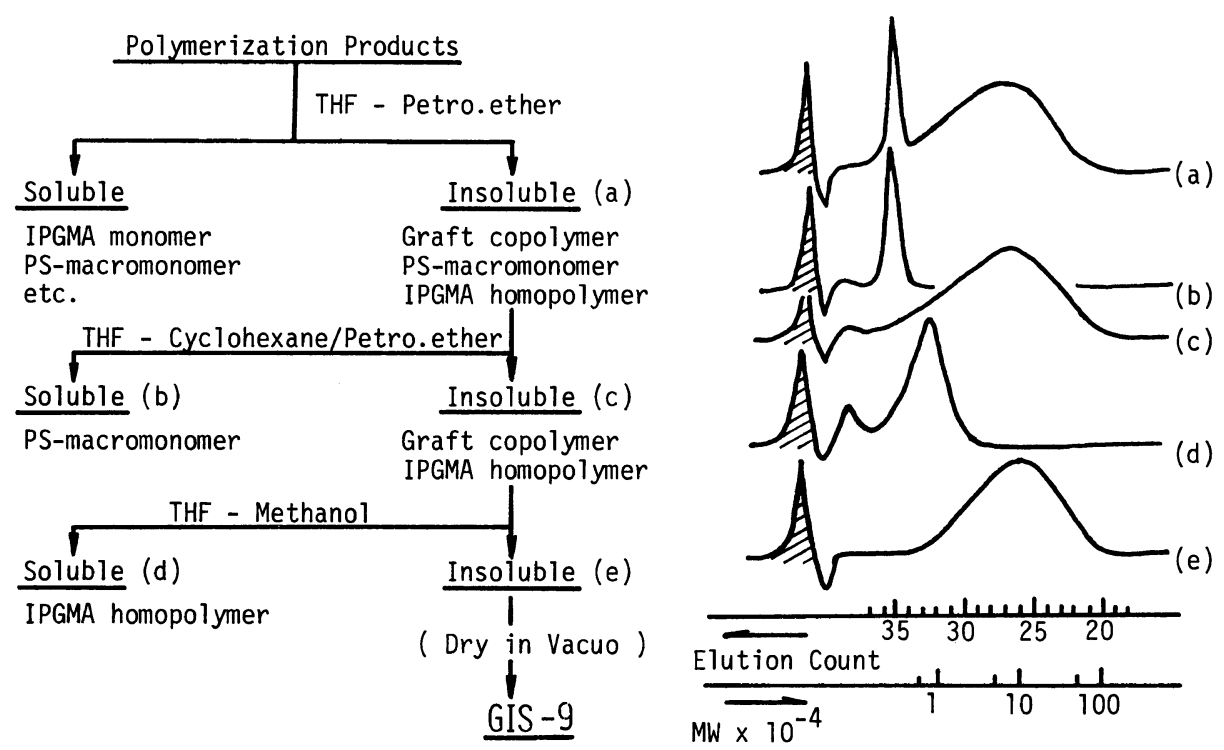

Scheme 3. Purification procedures of graft copolymers (GIS-9).

monomer with IPGMA comonomers were carried out with AIBN as an initiator and THF as a copolymerization solvent in glass ampoules degassed and sealed under vacuum at $60^{\circ} \mathrm{C}$ to obtain the poly(isopropylideneglyceryl methacrylate- $g$-styrene) (GIS) according to Scheme 2. Copolymerization products were precipitated in a large amount of petro. ether and reprecipitated and extracted care- fully with THF, cyclohexane, petro. ether and methanol to remove unreacted macromonomers, oligomers, and IPGMA homopolymers according to Scheme 3. The acidic hydrolysis of the acetal groups of GIS was carried out with $85 \%$ trifluoroacetic acid aqueous solution for 5-6 hours at room temperature to obtain poly(2,3-dihydroxypropyl methacrylate- $g$-styrene) (GDS). These graft copolymers 
were characterized by ${ }^{1} \mathrm{H}$ NMR, IR, elemental analysis and GPC. ${ }^{1} \mathrm{H}$ NMR spectra and IR spectra were taken with a JEOL JNM PMX-60 (60 MHz) and a JASCO IRA-1, respectively. GPC was carried out with a ToyoSoda HLC-802UR.

Preparation and Characterization of Hydrogels

$5 \mathrm{w} / \mathrm{v} \%$ of GDS graft copolymer solutions were prepared with THF-methanol mixed solvent and the solutions were poured onto flat Teflon plates. Then the solvent was evaporated gradually at room temperature to obtain the film specimens. The film thickness was $c a$. $75 \mu \mathrm{m}$ and the specimens with larger thickness of $c a .150 \mu \mathrm{m}$ and $250 \mu \mathrm{m}$ were also prepared for measuring the oxygen permeability coefficient of hydrogels.

For making electron microscopic observations of the microphase separated structure, film specimens were stained in an aqueous solution of osmium tetraoxide $\left(\mathrm{O}_{\mathrm{s}} \mathrm{O}_{4}\right)$ for 24 hours at room temperature and subsequently cut into ribbon shape, embedded in epoxy resin, trimmed, and stained again with $\mathrm{O}_{\mathrm{s}} \mathrm{O}_{4}$ aqueous solution at $50^{\circ} \mathrm{C}$ for 24 hours. Then the stained specimens were cut normally to the film surface to a thickness about $40-50 \mathrm{~nm}$ by a Dupont diamond knife using a Sorvall MT2B microtome. A JEM-100CX electron microscope of JEOL was used to obtain the electron microscopic photograph.

The film specimens were immersed into twice distilled water at $30^{\circ} \mathrm{C}$ and other temperatures for several days to prepare stable hydrogels with the equilibrium water content. The swelling degree $(Q)$ and the water content $(H)$ are calculated by $Q=W_{\text {wet }} / W_{\text {dry }} \times 100$ and $H=\left(W_{\text {wet }}-W_{\text {dry }}\right) / W_{\text {wet }} \times 100$, where $W_{\text {wet }}$ is the weight of hydrogel at swollen state and $W_{\text {dry }}$ is that at unswollen state.

The SAXS intensity distributions from the specimens were measured with a water-cooled copper-anode tube powered by a JEOL X-ray generator (Model DX-GE-2D, operated at $45 \mathrm{kV}$ and $35 \mathrm{~mA}$ ) at room temperature $\left(25 \pm 2^{\circ} \mathrm{C}\right) .^{24-26}$ The intensity was measured with a Kratky U-slit camera of Anton Paar Co. and a scintillation counter provided with a Nickel filter and a differential pulse-height analyzer (Philips PW4280/01) to receive the $0.154 \mathrm{~nm} \mathrm{Cu}-K_{\alpha}$ radiation. The widths of the entrance and counter slits were 100 and $250 \mu \mathrm{m}$ respectively, and the distance between sample and the plane of registration was $210 \mathrm{~mm}$. The optical alignment procedure of the camera was carried out according to the instruction manual from Anton Paar Co. Film specimens in the swollen state were completely rapped with Aluminum foil to prevent the evaporation of water from the sample during the measurement. The measured intensity was corrected for scattering and absorption of air and $\mathrm{Al}$ foil. The correction of finite slit height and width was carried out according to Glatter's program $^{26}$ to yield the desmeared scattering intensity which would be obtained with a pinhole X-ray source. The calculation was carried out at Nagoya University Computer Center, using a Facom 230-60/75.

The oxygen permeability coefficients through hydrogels in water were measured at $30^{\circ} \mathrm{C}$ with several specimens having different thickness by using an Oxygen Permeability Meter of Rikaseiki Kogyo Co., Ltd. with a silver anode and a platinum cathode. Hydrogel specimens circularly cut with $18 \mathrm{~mm}$ diameter were set at close contact with the cathode and immersed into distilled water which contained saturated oxygen gas by bubbling. An electric current caused by the reaction of oxygen permeating hydrogels with the electrode was measured with an ampere meter. The oxygen permeability coefficient was calculated from a saturated value of the electric current.

\section{RESULTS AND DISCUSSION}

\section{Preparation of Poly(2,3-dihydroxypropyl methacrylate-g-styrene) \\ Uniform molecular weights of St macro- monomers were prepared by the living}


Synthesis and Characterization of Hydrogels from Graft Copolymers

Table I. Preparation of graft copolymers ${ }^{\mathrm{a}}$

\begin{tabular}{|c|c|c|c|c|c|c|c|c|c|}
\hline \multirow{2}{*}{$\begin{array}{l}\text { Sample } \\
\text { code }\end{array}$} & \multicolumn{2}{|c|}{ Feed composition $/ w t \%$} & \multirow{2}{*}{$\begin{array}{l}\text { Dilution } \\
\text { rate } \\
\text { (THF) }\end{array}$} & \multirow{2}{*}{$\frac{\text { AIBN }}{w t^{\circ} \%}$} & \multicolumn{3}{|c|}{ Macromonomer } & \multirow{2}{*}{$\frac{\text { Polym. time }}{\text { day }}$} & \multirow{2}{*}{$\frac{\text { Yield }}{\%}$} \\
\hline & Macromonomer & Comonomer & & & $\bar{M}_{\mathrm{n}} \times 10^{-3 \mathrm{~b}}$ & $\bar{M}_{w} / \bar{M}_{n}$ & $f^{c}$ & & \\
\hline GIS-1 & 50.0 & 50.0 & 6.2 & 0.030 & 7.23 & 1.06 & 0.7 & 4.0 & 53.8 \\
\hline GIS-2 & 50.0 & 50.0 & 6.2 & 0.015 & 7.23 & 1.06 & 0.7 & 4.0 & 55.0 \\
\hline GIS-4 & 25.0 & 75.0 & 3.6 & 0.030 & 3.13 & 1.10 & 0.8 & 4.0 & 88.0 \\
\hline GIS-5 & 25.0 & 75.0 & 2.8 & 0.068 & 1.88 & 1.10 & 0.6 & 5.0 & 76.9 \\
\hline GIS-6 & 25.0 & 75.0 & 2.8 & 0.068 & 6.80 & 1.06 & 0.8 & 5.0 & 87.9 \\
\hline GIS-7 & 25.0 & 75.0 & 2.8 & 0.068 & 3.35 & 1.16 & 0.7 & 5.0 & 88.0 \\
\hline GIS-8 & 25.0 & 75.0 & 3.1 & 0.015 & 5.37 & 1.05 & 0.8 & 5.8 & 80.0 \\
\hline GIS-9 & $33: 3$ & 66.7 & 3.1 & 0.015 & 5.37 & 1.05 & 0.8 & 5.8 & 75.0 \\
\hline GIS-10 & 50.0 & 50.0 & 3.1 & 0.015 & 5.37 & 1.05 & 0.8 & 5.8 & 60.7 \\
\hline GIS-11 & 75.0 & 25.0 & 3.1 & 0.015 & 5.37 & 1.05 & 0.8 & 5.8 & 48.0 \\
\hline GIS-12 & 50.0 & 50.0 & 2.5 & 0.016 & 22.47 & 1.29 & 0.8 & 8.0 & 48.7 \\
\hline GIS-13 & 80.0 & 20.0 & 2.5 & 0.016 & 22.47 & 1.29 & 0.8 & 8.0 & 33.5 \\
\hline GIS-15 & 50.0 & 50.0 & 2.0 & 0.015 & 12.40 & 1.06 & 0.9 & 4.0 & 78.1 \\
\hline
\end{tabular}

a Copolymerization was carried out at $60^{\circ} \mathrm{C}$ with AIBN as an initiator.

b Determined from GPC using calibrations with standard polystyrene samples.

c End functionality was estimated from the titration in THF solution with $\mathrm{KOH}$.

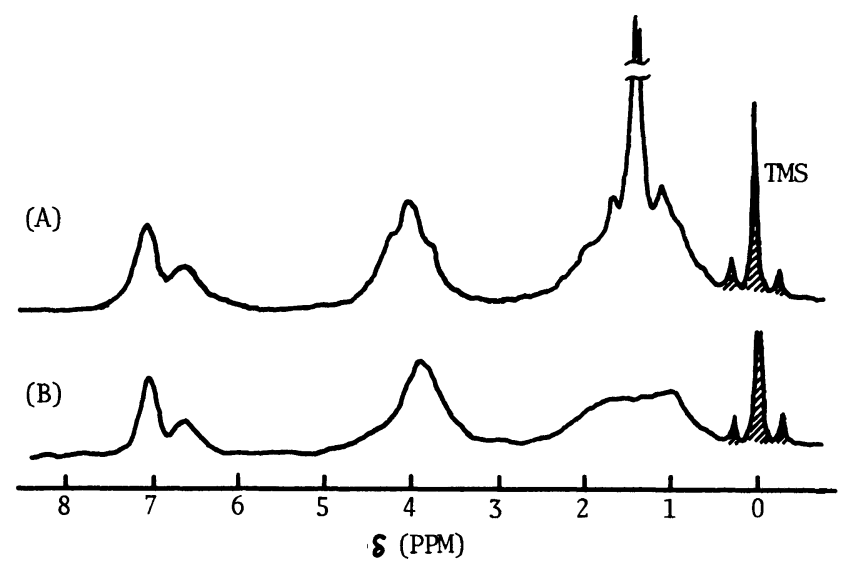

Figure 1. ${ }^{1} \mathrm{H}$ NMR spectra of the graft copolymer GIS-6(A) before and (B) after the hydrolysis reaction. (A), in chloroform $\left(d_{1}\right)$; (B), in methanol $\left(d_{4}\right)$-tetrahydrofuran $\left(d_{8}\right)$.

anionic polymerization of St monomers terminated by the coupling reaction with carbon dioxide followed by reaction with glycidyl methacrylate to obtain a methacryloxyl end group at one end. The content of the terminal carboxyl group of the St prepolymer determined by titrations with $\mathrm{KOH}$ was in the range of 0.7 to 0.9 . The reaction of the terminal carboxyl group with glycidyl meth- acrylate gave the St macromonomer with $80-90 \%$ reaction yield.

The details of the preparation condition of graft copolymers are shown in Table I with the characteristics of the obtained St macromonomers. THF was used as the copolymerization solvent to keep the reaction systems homogeneous since GIS were soluble in tetrahydrofuran (THF). Copolymerization 
products were purified by careful extraction-reprecipitation procedure with the selective solvent for both components according to Scheme 3. Each step of the purification procedure was monitored with GPC. The procedures were continued until the sharp peak of the unreacted St macromonomer at the lower molecular side of the produced graft copolymer main peak became negligible and finally the extraction of IPGMA homopolymer was carried out with methanol.

Since the distinct phenyl proton peaks of PSt branch of the purified graft copolymers can be observed in ${ }^{1} \mathrm{H} \mathrm{NMR}$, as shown in Figure 1, the formation of the graft copolymer can be fairly confirmed. Conversion of GIS to GDS was carried out by the acid hydrolysis reaction. The conversion was confirmed by ${ }^{1} \mathrm{H}$ NMR spectra as shown in Figure 1, where the spectra were measured (A) before and (B) after the hydrolysis reaction. It can be seen from the figure that the distinct double peaks of the isopropylidene proton observed in (A) disappears completely in (B) and the integral intensity ratio of the ester proton $(c a .3 .9 \mathrm{ppm})$ to the phenyl proton of St monomer units ( $c a$. $7.0 \mathrm{ppm}$ ) is almost constant. This indicates that the hydrolysis reaction proceeded quantitatively and was limited to the acetal groups. IR spectra of GDS also showed a distinct broad $\mathrm{OH}$ band around $3400 \mathrm{~cm}^{-1}$ indicating the formation of 2,3-dihydroxypropyl groups. Furthermore, the obtained GDS graft copolymers were soluble in THF-methanol mixed solvent but insoluble in pure THF. The characteristics of GDS are summarized in Table II.

The number of PSt branches per graft copolymer $(n)$ and the number average molecular weight of the backbone segment between the two adjoining branches $\left(\bar{M}_{n}^{\mathrm{s}}\right)$ in Table II were calculated by $n=\bar{M}_{n} \times W_{\mathrm{s}} / \bar{M}_{n}{ }^{\mathrm{b}}$ and $\bar{M}_{n}^{\mathrm{s}}=\bar{M}_{n} \times\left(1-W_{\mathrm{s}}\right) / n$ respectively, where $\bar{M}_{n}$ is the number average total molecular weight of GDS graft copolymer, $\bar{M}_{n}{ }^{\text {b }}$ is the number average molecular weight of the PSt

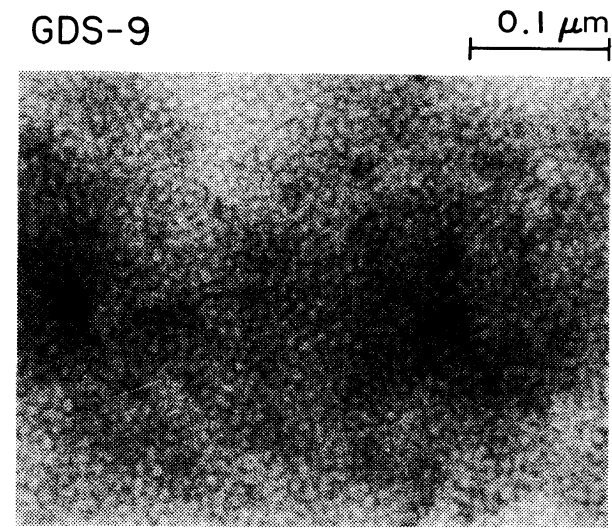

Figure 2. Typical transmission electron micrograph of graft copolymer cast from methanol-THF (1:1) mixed solvent; stained with $\mathrm{O}_{\mathrm{s}} \mathrm{O}_{4}$ aqueous solution.

branch, and $W_{\mathrm{s}}$ is the weight fraction of $\mathrm{St}$ component in the graft copolymer. The St content in the graft copolymer ranges from 0.09 to $0.55 \bar{M}_{n}^{\text {b }}$ ranges from $1.9 \times 10^{3}$ to $22.3 \times 10^{3}$ and $\bar{M}_{n}{ }^{\text {s }}$ ranges from $6.5 \times 10^{3}$ to $67.4 \times 10^{3}$.

Figure 2 shows a typical electron micrograph of graft copolymer (GDS-9) cast from methanol-THF equivolume mixture and stained with $\mathrm{O}_{\mathrm{s}} \mathrm{O}_{4}$. 2,3-Dihydroxypropyl methacrylate (DHPMA) segments in GDS graft copolymers can be stained with $\mathrm{O}_{\mathrm{s}} \mathrm{O}_{4}$ as in the case of HEMA component, ${ }^{13,15}$ so that the dark continuous phase in the figure corresponds to the DHPMA phase and the bright spherical phase corresponds to the St phase respectively. It can be seen in the figure that the spherical microdomain structure is considerably regular and consistent with the volume consideration ( $\mathrm{St} \mathrm{wt} \%$ of GDS-9 is 17.0), although the image contrast of two phases is rather weak. The average radius of St domains is about $50 \AA$, where the molecular weight of St branch is 5400 .

\section{Swelling Behaviors of Poly(2,3-dihydroxy- propyl methacrylate-g-styrene)}

Solution cast films of GDS swelled immediately when they were immersed into distil- 


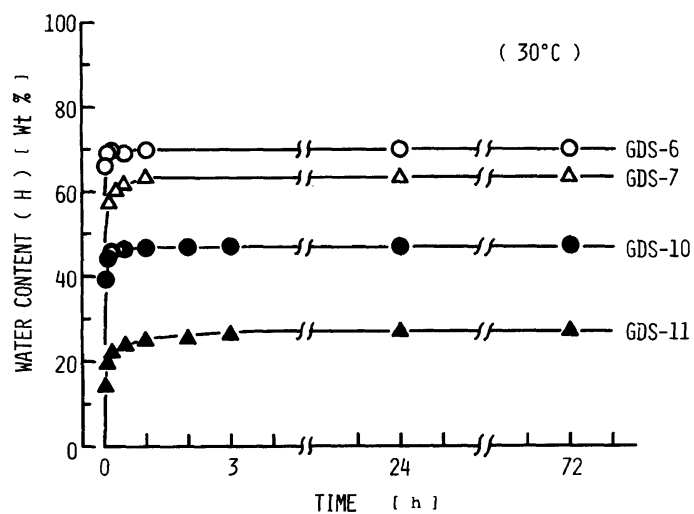

Figure 3. Changes of water content with incubation time at $30^{\circ} \mathrm{C}$.

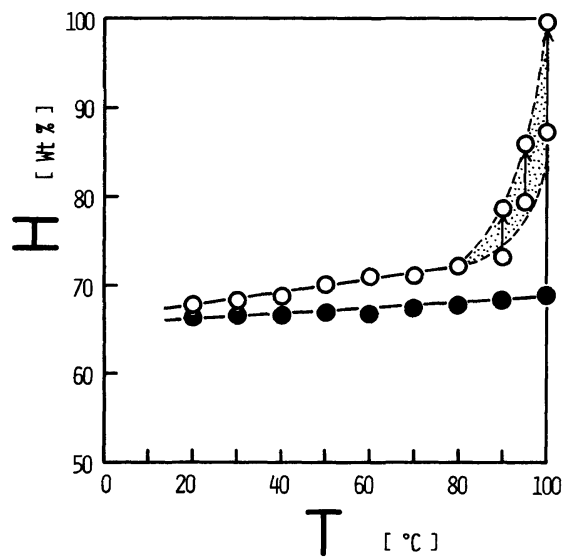

Figure 4. Effect of temperature on the water content $(H)$ of graft copolymer GDS-9 cast from methanolTHF (1:1) mixture.

led water as shown in Figure 3, where typical water content versus time curves are shown. In this figure, water content in each sample rapidly increases in the first 10 minutes and goes up to the saturated value within several hours irrespective of equilibrium water content.

The obtained hydrogels were soft and stable and also showed good mechanical strength enough to endure the handling of the following measurements. Figure 4 shows the effect of temperature on the water content $(H)$ of particular GDS-9 specimen (-O-) and poly $(2,3-$ dihydroxypropyl methacrylate) (PDHPMA) homopolymer crosslinked with ethyleneglycoldimethacrylate (EDMA) (- - ), in which the $H$ values of both samples increase with increasing in temperature with almost the same slope. The hydrogel of GDS-9 was stable except in the temperature range where the glass transition effect of PSt domain was observed. That is, the equilibrium water content of GDS-9 was obtained in a few hours of incubation time below $80^{\circ} \mathrm{C}$ and the size and weight of the hydrogel did not change. However, above $80^{\circ} \mathrm{C}$ the water content kept increasing with incubation time even after one week. On the other hand, the crosslinked PDHPMA was almost stable even near $100^{\circ} \mathrm{C}$.

The appearance of hydrogels and the equilibrium water content $(H)$ and the equilibrium swelling degree $(Q)$ of hydrogels, cast from methanol-THF equivolume mixture, are shown in Table II. All of these hydrogels in dry state (as cast specimen) were transparent, whereas some of them became slightly translucent in the swollen state. This may be partially related to the water-induced-heterogeneity (syneresis) and also partially related to the degree of purification of each graft copolymer. However, the reason is not clear at present. The water content of hydrogels at $30^{\circ} \mathrm{C}$ in Table II varies from 27.3 to $81.8 \%$ with the graft copolymer structure, while that of PSt homopolymer was $1.7 \%$ under the same condition.

\section{Effect of Graft Copolymer Structure}

Figure 5 shows the relationship between the water content and weight fraction of St component in the graft copolymers, where it is seen that $H$ increases with decreasing in $W_{\mathrm{s}}$. However, there is considerable scattering of plotted data in the figure. In Figure 6, $Q$ (and $H$ ) is replotted as a function of $\bar{M}_{n}{ }^{\text {s }}$. It is seen from the figure that the correlation between $Q$ (or $H$ ) and $\bar{M}_{n}^{\text {s }}$ is much better than that between $H$ and $W_{\mathrm{s}}$ in Figure 5. It is also seen that $\log Q$ versus $\log \bar{M}_{n}{ }^{\mathrm{s}}$ plots fall on almost a 
Table II. Characteristics of graft copolymers and hydrogel membranes

\begin{tabular}{|c|c|c|c|c|c|c|c|c|c|c|c|}
\hline \multirow{3}{*}{$\begin{array}{l}\text { Sample } \\
\text { code }\end{array}$} & \multicolumn{3}{|c|}{$\bar{M}_{n} \times 10^{-3 \mathrm{a}}$} & \multirow{3}{*}{$\begin{array}{l}\text { Number } \\
\text { of } \\
\text { branches }\end{array}$} & \multirow{3}{*}{$\begin{array}{c}\bar{M}_{n}^{\mathrm{s}} \\
\times 10^{-3}\end{array}$} & \multirow{2}{*}{\multicolumn{2}{|c|}{$\begin{array}{c}\text { Composition }^{b} \\
\text { wt } \%\end{array}$}} & \multicolumn{4}{|c|}{ Hydrogel properties } \\
\hline & \multirow{2}{*}{ Total } & \multirow{2}{*}{$\begin{array}{l}\text { DHPMA- } \\
\text { backbone }\end{array}$} & \multirow{2}{*}{$\begin{array}{c}\text { PSt- } \\
\text { branch }\end{array}$} & & & & & $H$ & $Q$ & & \\
\hline & & & & & & PSt & DHPMA & $\%$ & $\%$ & $\times 10^{9}$ & Appearance \\
\hline GDS-1 & 41.4 & 31.3 & 7.23 & 1.4 & 22.3 & 24.5 & 75.5 & 70.1 & 334 & - & t.1. \\
\hline GDS-2 & 49.4 & 37.6 & 7.23 & 1.7 & 22.1 & 24.3 & 75.7 & 60.9 & 256 & - & t.l. \\
\hline GDS-4 & 66.0 & 51.6 & 3.13 & 4.6 & 11.2 & 21.6 & 78.4 & 43.9 & 178 & 1.57 & t.l. \\
\hline GDS-5 & 75.7 & 68.4 & 1.88 & 3.9 & 17.5 & 9.6 & 90.4 & 66.9 & 302 & 3.42 & t.p. \\
\hline GDS-6 & 48.8 & 39.9 & 6.80 & 1.4 & 28.5 & 19.0 & 81.0 & 65.6 & 291 & 2.95 & t.l. \\
\hline GDS-7 & 35.4 & 30.0 & 3.35 & 1.6 & 18.8 & 15.2 & 84.8 & 63.3 & 272 & 2.07 & t.l. \\
\hline GDS-8 & 89.9 & 78.6 & 5.37 & 2.1 & 37.4 & 12.3 & 87.7 & 75.0 & 400 & 3.37 & t.p. \\
\hline GDS-9 & 101.1 & 83.9 & 5.37 & 3.2 & 26.2 & 17.0 & 83.0 & 68.4 & 316 & 3.04 & t.p. \\
\hline GDS-10 & 78.8 & 52.5 & 5.37 & 4.9 & 10.7 & 33.4 & 66.6 & 47.0 & 189 & 1.53 & t.1. \\
\hline GDS-11 & 79.6 & 43.6 & 5.37 & 6.7 & 6.5 & 45.1 & 54.9 & 27.3 & 138 & 0.81 & t.p. \\
\hline GDS-12 & 150.2 & 112.6 & 22.47 & 1.7 & 67.4 & 25.0 & 75.0 & 81.8 & 549 & 5.76 & t.l. \\
\hline GDS-13 & 108.7 & 49.4 & 22.47 & 2.6 & 18.7 & 54.6 & 45.4 & 46.8 & 188 & 1.63 & t.l. \\
\hline GDS-15 & 69.5 & 36.0 & 12.40 & 2.7 & 13.3 & 48.3 & 51.7 & 40.4 & 168 & 1.80 & t.p. \\
\hline
\end{tabular}

a Determined from GPC equipped with light scattering detector.

b Determined from ${ }^{1} \mathrm{H}$ NMR spectra and elemental analysis.

c t.p. = transparent; t.l. = translucent.

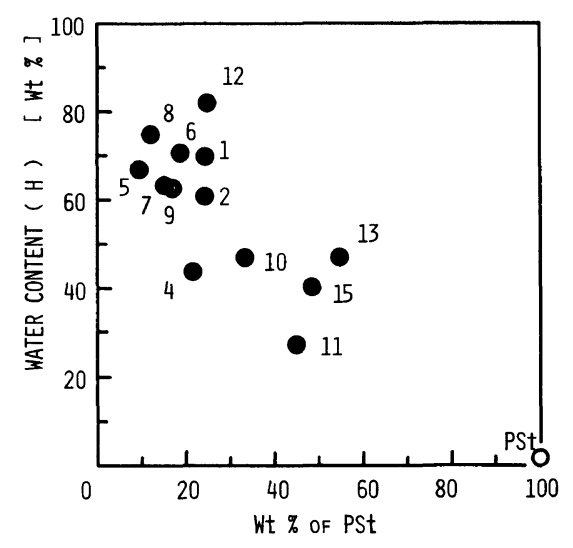

Figure 5. Relation between water content $(H)$ and St content of graft copolymers $\left(W_{\mathrm{s}}\right)$ cast from methanolTHF $(1: 1)$ mixture. Water content were measured at $30^{\circ} \mathrm{C}$. The number in the figure indicates the sample code number in Table II.

straight line with the slope of about 0.6 although there is still some degree of scattering of data. This result is consistent with Flory's theory for the swelling behaviour of network polymers where $Q \sim M_{\mathrm{c}}^{3 / 5}$ is predicted $\left(M_{\mathrm{c}}\right.$, the molecular weight of the polymer segment be-

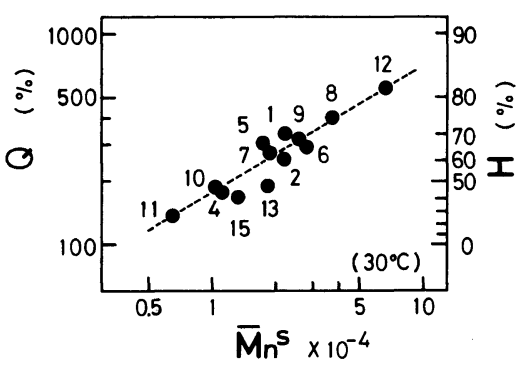

Figure 6. Swelling degree $(Q)$ of graft copolymers plotted against molecular weight of the backbone segment between two adjoining PSt branches $\left(\bar{M}_{n}^{\text {s }}\right)$.

tween crosslinking points). ${ }^{27}$ This indicates that only hydrophilic 2,3-dihydroxypropyl methacrylate (DHPMA) backbone segments take part in swelling and PSt domains do not affect swelling behaviour and act just as rigid crosslinking points in hydrogels. This shows that the swelling behaviour of hydrogels is well controlled by $\bar{M}_{n}^{\text {s }}$, i.e., the molecular weight of the backbone segment between two adjoining branches in the graft copolymer.

It should be noted that $H$ also increases with decrease in the number of branches per graft 
Table III. Microdomain structure of poly(2,3-dihydroxypropyl methacrylate- $g$-styrene) hydrogels

\begin{tabular}{|c|c|c|c|c|c|c|c|c|}
\hline \multirow{3}{*}{$\begin{array}{l}\text { Sample } \\
\text { code }\end{array}$} & \multicolumn{3}{|c|}{$S_{\max }$ in SAXS curve ${ }^{\mathrm{b}}$} & \multicolumn{3}{|c|}{ Domain structure/nm } & \multirow{3}{*}{$N$} & \multirow{3}{*}{$\left(D_{\text {wet }} / D_{\text {dry }}\right)^{3}$} \\
\hline & \multicolumn{2}{|c|}{ Interdomain } & \multirow{2}{*}{$\frac{\text { Intradomain }}{\text { Wet }}$} & \multicolumn{2}{|c|}{$D$} & \multirow{2}{*}{$R$} & & \\
\hline & Dry & Wet & & Dry & Wet & & & \\
\hline GDS-8 & 0.0297 & 0.0173 & 0.0900 & 21.2 & 36.3 & 6.4 & 130 & 5.02 \\
\hline GDS-9 & 0.0295 & $0: 0188$ & 0.0800 & 21.3 & 33.5 & $\begin{array}{l}7.2 \\
(5.0 \pm 1.0)^{\mathrm{a}}\end{array}$ & 183 & 3.92 \\
\hline GDS-10 & 0.0316 & 0.0277 & 0.0876 & 19.9 & 22.6 & 6.6 & 141 & 1.47 \\
\hline GDS-15c & 0.0232 & 0.0214 & 0.0623 & 27.0 & 29.4 & - & - & 1.29 \\
\hline
\end{tabular}

a Estimated from electron microscopy.

b $S$, the scattering vector $(=4 \pi / \lambda \cdot \sin (\theta / 2)$, where $\theta$ is the scattering angle); $D$, interdomain distance; $R$, radius of styrene domain; $N$, number of branches per PSt-domain.

c Lamellar microdomain structure.

copolymer ( $n$ ) and $H$ approaches $100 \%$ (or $Q$ approaches infinity) near $n$ equals unity where graft copolymers cannot form a network structure but dissolve into water. However, in regard to the St branch length, it does not influence the swelling behaviour directly, although it might affect the size of PSt microdomains and thus the mechanical strength against the swelling pressure as shown later.

\section{Effects of the Microphase Separated Structure}

The microdomain structure of graft copolymers as well as block copolymers can be wellcharacterized by measuring of SAXS intensity distribution from them. ${ }^{28,29}$ In Table III, the results of SAXS measurement on the microdomain structure of particular samples of GDS-8, GDS-9, GDS-10, and GDS-15 are summarized. There were at least two distinct scattering maxima associated with the interparticle interference and a broad scattering maximum associated with the intraparticle interference from isolated PSt domains in all scattering curves of these samples. The scattering peak position of the interparticle interference from PSt microdomains were assumed to shift toward a short angle direction by swelling, whereas the peak position of intraparticle interference from the isolated PSt microdomain was assumed to be unchanged by swelling of hydrogels.

The domain distance $(D)$ between PSt microdomains was calculated from the scattering angle $(\theta)$ of the 1st-interparticle interference maximum by using Bragg's equation:

$$
D=\lambda / 2 \sin \left(\theta_{\max } / 2\right)
$$

and the radius of PSt domains $(R)$ was calculated from the angle of the scattering maximum from the isolated spherical PSt domain by using:

$$
\begin{aligned}
(4 \pi R / \lambda) \sin \left(\theta_{\max , n} / 2\right)= & 5.765,9.10,12.3 \\
& (\text { for } n=1,2,3)
\end{aligned}
$$

The number of PSt branches per PSt domain $(N)$ in the table was calculated from $R$ with:

$$
4 \pi R^{3} / 3=N v_{\mathrm{st}}=N \rho N_{\mathrm{A}} / \bar{M}_{n}{ }^{\mathrm{b}}
$$

by assuming the spherical microdomain and the same segment density in the domain as bulk, where $v_{\mathrm{st}}$ is the molecular volume of a polystyrene branch, $\rho$ is the bulk density $(1.052$ $\left.\mathrm{g} \mathrm{ml}^{-1}\right)$, and $N_{\mathrm{A}}$ is avogadro's number. $\left(D_{\text {wet }} /\right.$ $\left.D_{\mathrm{dry}}\right)^{3}$ in the table corresponds to the swelling degree measured by the change in the domain distance, where $D_{\text {wet }}$ is the domain distance between PSt microdomains in the swollen state, and $D_{\text {dry }}$ is that in the dry state.

Figure 7 shows changes in the interdomian 


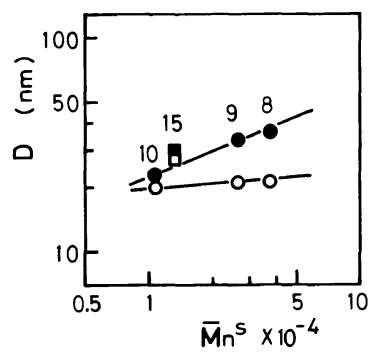

Figure 7. Change in the domain distance $D$ of graft copolymers of GDS-8, GDS-9, GDS-10, and GDS-15 by swelling as a function of $\bar{M}_{n}^{\text {s }}$. Open symbols correspond to dry state and closed symbols correspond to swollen state. Square symbols represent lamellar morphology.

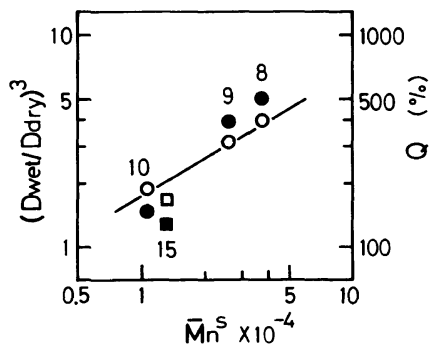

Figure 8. Plots of $\left(D_{\text {wet }} / D_{\text {dry }}\right)^{3}$ and $Q$ against $\bar{M}_{n}^{\text {s. }}$ : Closed symbols, $\left(D_{\text {wet }} / D_{\text {dry }}\right)^{3}$ versus $\bar{M}_{n}^{\text {s; }}$ open symbols, $Q$ versus $\bar{M}_{n}^{\text {s. }}$. Straight line corresponds to $Q \sim\left(\bar{M}_{n}^{\mathrm{s}}\right)^{3 / 5}$.

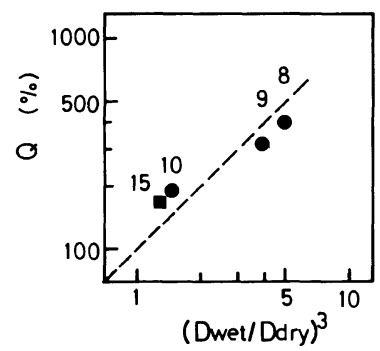

Figure 9. Relation between $Q$ and $\left(D_{\text {wet }} / D_{\text {dry }}\right)^{3}$.

distance by swelling as a function of $\bar{M}_{n}^{\mathrm{s}}$. The microdomain structures of GDS-8, GDS-9, and GDS-10 are considered to be spherical whereas that of GDS-15 is considered to be a lamellar structure from the SAXS intensity profile and volume consideration. In this figure, graft copolymers have the same branch length $\left(\bar{M}_{n}\right.$ is 5400) except for GDS-15, but have different branch numbers and thus different $\bar{M}_{n}$ s (see Table II). As seen in the figure,

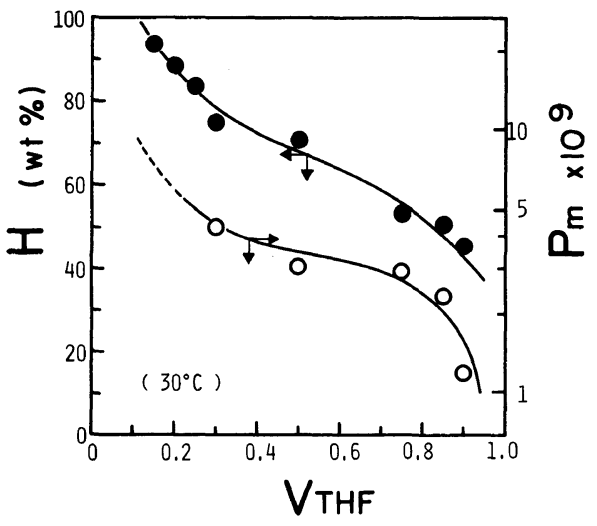

Figure 10. Influence of composition of cast solvent (methanol-THF mixture) on oxygen permeability coefficient $\left(P_{\mathrm{m}}, O\right)$ in $\left[\mathrm{ml}(\mathrm{STP}) \mathrm{cm} \mathrm{cm}^{-2} \mathrm{~s}^{-1} \mathrm{~cm} \mathrm{Hg}^{-1}\right]$ and water content $(H, \bigcirc)$ of GDS-6 hydrogel measured at $30^{\circ} \mathrm{C}$.

the increment of the domain distance by swelling becomes larger with increasing of $\bar{M}_{n}^{\mathrm{s}}$ as expected. However, it is not clear at present as to the reason for the small dependence of $D$ in the dry state on $\bar{M}_{n}^{\text {s }}$. Figure 8 shows relation between $\left(D_{\text {wet }} / D_{\text {dry }}\right)^{3}$ and $\bar{M}_{n}^{\text {s }}$ together with $Q$ versus $\bar{M}_{n}^{\text {s }}$. As seen in the figure, both $\left(D_{\text {wet }} /\right.$ $\left.D_{\text {dry }}\right)^{3}$ and $Q$ increase with increasing of $\bar{M}_{n}^{\mathrm{s}}$. In Figure 9 the relation between $\left(D_{\text {wet }} / D_{\text {dry }}\right)^{3}$ and $Q$ is shown. It is seen that $\left(D_{\text {wet }} / D_{\mathrm{dry}}\right)^{3}$, the swelling degree in colloidal order, is well consistent with the macroscopic swelling degree of $Q$, indicating that swelling behavior of graft copolymers is also well correlated with and controlled by the microdomain structure in the hydrogels.

It is well known that the microphase separated structure of block copolymers and graft copolymers depends on the quality of the cast solvent, in addition to their dependence on the molecular characteristics of copolymers such as the molecular weight and the composition. $^{31-35}$ This fact is known to be ascribed to the relative chain dimension of each component in the cast solution which determines the morphology of the domain structure. In this study, a THF-methanol equivolume mixture was used as the cast solvent to prepare 


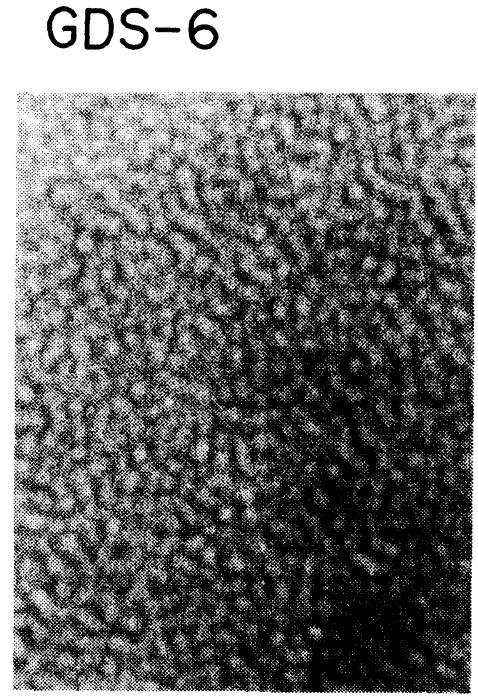

(a)
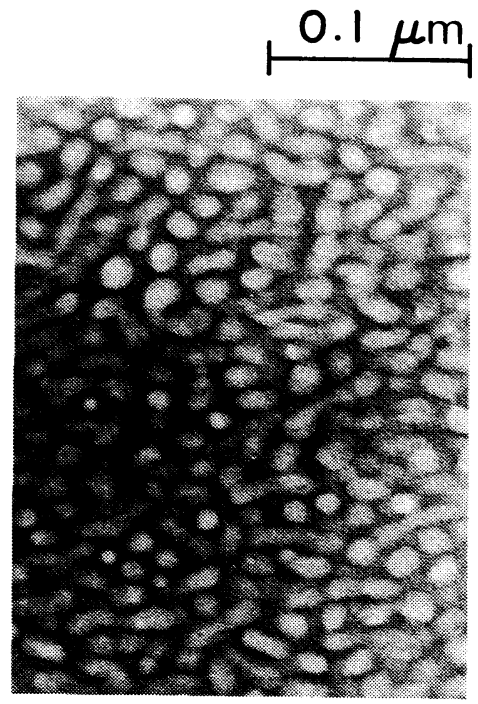

(b)

Figure 11. Typical transmission micrographs of GDS-6 cast from methanol-THF mixed solvent: THF content (a) $90 \mathrm{v} / \mathrm{v} \%$, (b) $20 \mathrm{v} / \mathrm{v} \%$.

hydrogels in the preceding section because the synthesized graft copolymers did not dissolve in both THF and methanol single solvent. In this case, methanol is a preferential solvent for DHPMA segments while THF is a preferential solvent for PSt branches. Figure 10 shows the influence of the mixed ratio of the THF-methanol mixture in solvent casting on the water content and the oxygen permeability $\left(P_{\mathrm{m}}\right)$ of hydrogels. The details of the oxygen permeability through hydrogels will be discussed later. It is seen in the figure that $H$ and also $P_{\mathrm{m}}$ are influenced by the composition of the cast solvent both of which decrease with increasing THF content in the mixed solvent. This result can be understood with respect to change in the microphase separated structure with change in quality of cast solvents. With increasing of THF content in the cast solvent, PSt branches in the graft copolymer can expand more in the solution. This causes increase in volume fraction of PSt domain in the cast solution and then causes the PSt phase to be more continuous in the cast specimen after the evaporation of the solvent. Therefore, hydrogels prepared from THF-rich solvent might encounter much more resistance of PSt segments to swell in water.

Figure 11 shows typical transmission electron micrographs of GDS-6 cast from methanol-THF mixed solvent of differnet compositions, THF content of which is $90 \mathrm{v} / \mathrm{v} \%$ in (a) and $20 \mathrm{v} / \mathrm{v} \%$ in (b). As seen in the figure, the polystyrene microdomains seem to have a tendency to connect with each other resulting in the formation of a rather continuous phase in Figure 11(a), whereas in Figure 11(b) both the PSt phase and PDHPMA phase are enlarged and the domain distance between PSt microdomains increase accompanied also with increase in irregularity of the microdomains compared with Figure 11(a). The increase of swelling at high methanol content in Figure 10 might be related to the increase in the domain distance in Figure 11(b), although the effect of the irregularity is not clear at present. 


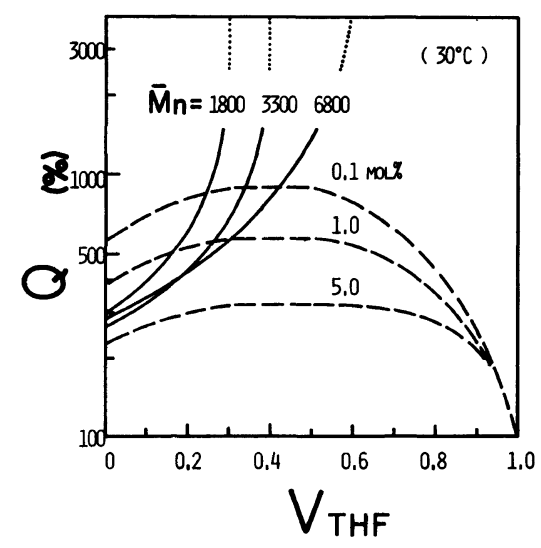

Figure 12. Influence of addition of THF to water on swelling behavior of graft copolymers of GDS-5, GDS7, GDS-6, and DHPMA homopolymer crosslinked with different amount of EDMA. Molecular weight of PSt branches of these graft copolymers are 1880, 3350, and 6800 , respectively. (measured at $30^{\circ} \mathrm{C}$ )

In Figure 12, the influence of plasticizing of PSt microdomain on the swelling behavior is shown with the hydrogel specimens prepared from THF-methanol equivolume mixture at $30^{\circ} \mathrm{C}$. In this figure hydrogels were immersed in water containing various amounts of THF and the swelling degree was measured as a function of THF content. Solid lines in the figure show the swelling degree of the gaft copolymers GDS-5, GDS-7, and GDS-6 the molecular weight of PSt branches of which are 1880,3350 , and 6800 , respectively. The broken lines show the degree of swelling of DHPMA homopolymers crosslinked with different amounts of ethyleneglycoldimethacrylate (EDMA). It is seen in the figure that the DHPMA segments expand and swell much more in the water-THF mixture than in the pure water. The maximum swellings are seen in the range from 0.3 to 0.5 of THF content and do not swell in pure THF. On the other hand, the swelling degree of the graft copolymer increases monotonically with increasing THF content in water until the gel collapses. The graft copolymer gel cannot be supported against swelling pressure beyond the collapse point which depends on the St branch length of the graft copolymer. That is, beyond this range the cohesive force between St segments becomes too weak to support PSt domains against the osmotic pressure because of the plasticizing effect of THF. The cohesive force fo PSt domain depends on the length of St branches. This aspect is very important in preparation of the ionic hydrogel of high swelling degree from graft copolymer where much more sufficient mechanical strength of the PSt domain will be required. ${ }^{23}$ There might exist a critical molecular weight of PSt branches for the formation of PSt domains as the crosslinking points to give sufficient mechanical strength in water, which is a function of the swelling tendency of the hydrophilic backbone segment.

\section{Oxygen Permeability through Hydrogels}

The oxygen permeability coefficient of hydrogels is one of the most important quantities to be measured for their biomedical applications. In this study, $P_{\mathrm{m}}$ of hydrogels in water were measured by the same method as used by Hosaka et al. ${ }^{30}$ That is, the electric current of steady state $\left(i_{\infty}\right)$ caused by the reaction of oxygen molecules through the hydrogel with the cathode was measured by using several specimens having different thicknesses $\left(l_{\mathrm{m}}\right)$. Then, $P_{\mathrm{m}}$ was calculated from the following equation with consideration of the effects of the boundary layer and the electrolytic solution layer.

$$
N F A P_{\mathrm{s}} / i_{\infty}=l_{\mathrm{m}} / P_{\mathrm{m}}+l_{\mathrm{b}} / P_{\mathrm{b}}+l_{\mathrm{e}} / P_{\mathrm{e}}
$$

where $F$, Faraday's constant, $N$, electron number $(=4), A$, contact area of the hydrogel membrane with the cathode $\left(=0.196 \mathrm{~cm}^{2}\right), P_{\mathrm{s}}$, oxygen gas partial pressure $(=760 \mathrm{mmHg}), l_{\mathrm{b}}$, $l_{\mathrm{e}}$, the thickness of the boundary layer and the electrolytic solution layer, $P_{\mathrm{b}}, P_{\mathrm{e}}$, the oxygen permeability coefficient of each corresponding layer. Measured $N F A P_{\mathrm{s}} / i_{\infty}$ values plotted $v e-$ rsus $l_{\mathrm{m}}$ fall on a straight line, the slope of which gives $P_{\mathrm{m}}{ }^{-1}$. The $P_{\mathrm{m}}$ values thus determined are also shown in Table II. $P_{\mathrm{m}}$ ranges from $0.81 \times$ 


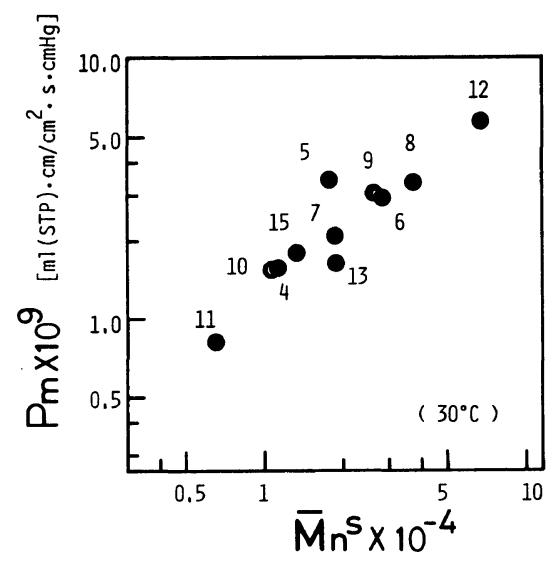

Figure 13. Oxygen permeability coefficient of hydrogels $\left(P_{\mathrm{m}}\right)$ in water measured at $30^{\circ} \mathrm{C}$ plotted against molecular weight of the backbone segment between two adjoining PSt branches $\left(\bar{M}_{n}^{s}\right)$.

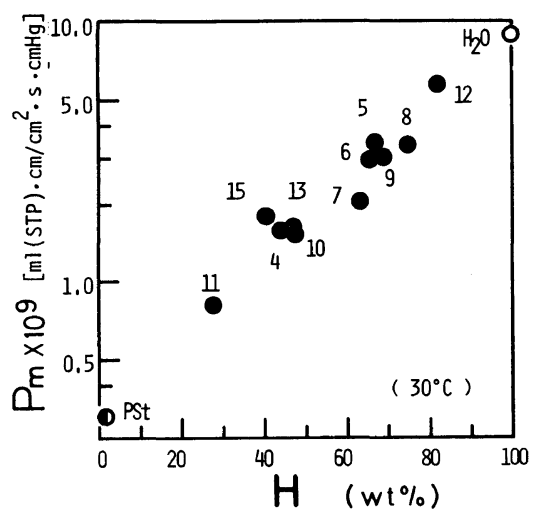

Figure 14. Relation between oxygen permeability coefficient $\left(P_{\mathrm{m}}\right)$ and water content $(H)$ of hydrogels including the values of PSt homopolymer and pure water at $30^{\circ} \mathrm{C}$.

$10^{-9}$ to $5.76 \times 10^{-9}\left[\mathrm{ml}(\mathrm{STP}) \mathrm{cm} \mathrm{cm}^{-2} \mathrm{~s}^{-1}\right.$ $\mathrm{cmHg}^{-1}$ ] depending on the water content of the hydrogel. These values are almost the same as the other hydrogels containing about $70 \%$ of water $\left(1.5 \times 10^{-9}-5.1 \times 10^{-9}\right) .^{30}$ Figure 13 shows the $P_{\mathrm{m}}$ variation with $\bar{M}_{n}{ }^{\text {s }}$ where $P_{\mathrm{m}}$ is well correlated to $\bar{M}_{n}{ }^{\mathrm{s}}$ as in the case of the swelling degree in Figure 6. The relationship between $P_{\mathrm{m}}$ and the water content $H$ is shown in Figure 14. It is seen in the figure that $P_{\mathrm{m}}$ values are directly related to the water content of the hydrogel and $\log P_{\mathrm{m}}$ versus $H$ plots fall on almost a straight line including the values of PSt and water. This result indicates that the oxygen permeability coefficient of the hydrogel is controlled by $\bar{M}_{n}^{\text {s }}$ via water content. Further, the $\bar{M}_{n}^{\text {s }}$ value itself also can be controlled by the feed ratio and other copolymerization conditions in the macromonomer method.

\section{CONCLUSIONS}

These experimental results are summarized as follows: 1) It was clearly demonstrated that well-defined graft copolymers can be obtained by using the macromonomer method. 2) The swelling degree of hydrogels is well-correlated with graft copolymer architecture, especially the molecular weight of the water soluble backbone segment between two adjoining branches of graft copolymers. 3) The microdomain structures of hydrogels are fairly regular and the macroscopic swelling behavior is well described with the change of the microdomain structure by swelling. 4) The oxygen permeability through the hydrogel in water is directly related to the swelling degree, and it was clearly shown that the oxygen permeability can be well controlled by the microdomain structure, the graft copolymer architecture, and copolymerization con ditions.

Acknowledgements. The authors are indebted to Mr. N. Toyoshima of Toyo Contact Lens Co., Ltd. for his kind supply of isopropylideneglyceryl methacrylate monomer. The authors are also deeply indebted to Dr. H. Sano of Mitsubishi Petrochemical Co., Ltd. for taking the electron micrographs in this work.

\section{REFERENCES}

1. O. Wichterle and D. Lim, Nature, 185, 117 (1960).

2. M. F. Refojo, J. Appl. Polym. Sci., 9, 3161 (1965); ibid., 9, 3417 (1965); M. F. Refojo and H. Yasuda, ibid., 9, 2425 (1965). 


\section{Y. TSUKAHARA et al.}

3. H. Yasuda, M. Gichin, and W. Stone, Jr., J. Polym. Sci., $A-1$, 4, 2913 (1966).

4. B. D. Ratner, P. K. Weatherby, and A. S. Hoffman, J. Appl. Polym. Sci., 22, 643 (1978).

5. M. Macret and G. Hild, Polymer, 23, 81 (1982), ibid., 23, 748 (1982).

6. S. Wisniewski and S. W. Kim, J. Membrane Sci., 6 , 299 (1980); ibid., 6, 309 (1980).

7. N. A. Peppas, R. Gurny, E. Doelker, and P. Buri, $J$. Membrane Sci., 7, 241 (1980).

8. S. Nagaoka, J. Suzuki, S. Kobayashi, M. Itoga, and H. Tanzawa, Maku (Membrane), 3, 59 (1978).

9. S. Hosaka, Maku (Membrane), 4, 155 (1979).

10. Y. Ikada, H. Iwata, F. Horii, T. Matsunaga, M. Suzuki, W. Taki, S. Yamagata, Y. Yonekawa, and H. Handa, J. Biomed. Mater. Res., 15, 697 (1981).

11. T. Matsuda, H. Takano, K. Hayashi, Y. Taenaka, S. Takaichi, M. Umezu, T. Nakamura, H. Iwata, and T. Nakatani, Trans. Am. Soc. Artif. Intern. Organs, 30, 353 (1984).

12. J. Pouchly, S. Benes, Z. Masa, and J. Biros, Makromol. Chem., 183, 1565 (1982).

13. S. Yamashita, K. Shibatani, K. Takakura, and S. Imai, Japan Patent 49-90743 (1974); Kobunshi Ronbunshu, 39, 187 (1982).

14. T. Nakashima, K. Takakura, and Y. Komoto, J. Biomed. Mater. Res., 11, 787 (1977).

15. M. Okano, M. Katayama, S. Mogi, and I. Shinohara, Nippon Kagaku Kaishi, 88 (1977).

16. Y. Tsukahara, A. Izumi, T. Hashimoto, and $\mathrm{H}$. Kawai, Polym. J., 14, 887 (1982).

17. Y. Yamashita, Appl. Polym. Symp., 36, 193 (1981); Y. Yamashita and Y. Tsukahara, "Modification of Polymers," C. E. Carraher, Jr. and J. A. Moore, Ed., Plenum, New York, N. Y., 1983, p 131. York, N. Y. 1983, p 131.

18. Y. Chujo, T. Shishino, Y. Tsukahara, and Y. Yamashita, Polym. J., 17, 133 (1985).

19. Y. Tsukahara, K. Kohno, H. Inoue, and Y.
Yamashita, Nippon Kagaku Kaishi, 1070 (1985).

20. K. Ito, N. Usami, and Y. Yamashita, Macromolecules, 13, 216 (1980); K. Ito, Y. Masuda, T. Shintani, T. Kitano, and Y. Yamashita, Polym. J., 15, 493 (1983).

21. Y. Kawakami, Y. Miki, T. Tsuda, R. N. A. Murthy, and Y. Yamashita, Polym. J., 14, 913 (1982).

22. Y. Yamashita, H. C. Tsai, and Y. Tsukahara, Makromol. Chem. Suppl., 12, 51 (1985).

23. H. C. Tsai, Y. Tsukahara, and Y. Yamashita, in preparation; Y. Tsukahara, H. C. Tsai, and Y. Yamashita, to be submitted.

24. Y. Muroga, I. Noda, M. Nagasawa, and T. Fukao, Biophys. Chem., 13, 97 (1981).

25. Y. Muroga, I. Noda, and M. Nagasawa, Macromolecules, 17, 1844 (1984); ibid., 18, 1576 (1985).

26. O. Glatter, J. Appl. Crystallogr., 7, 147 (1974).

27. P. J. Flory, "Principle of Polymer Chemistry," Cornell Univ. Press, Ithaca, New York, 1953.

28. T. Hashimoto, M. Shibayama, and H. Kawai, Macromolecules, 13, 1237 (1980); T. Hashimoto, M. Fujimura, and H. Kawai, ibid., 13, 1660 (1980). T. Hashimoto, "Polymer Alloys," Kobunshi Gakkai, Ed., Tokyo Kagaku Dohjin, Tokyo, 1981, p 47.

29. M. Shibayama, T. Hashimoto, and H. Kawai, Macromolecules, 16, 16 (1983).

30. S. Hosaka, Y. Adachi, and H. Tanzawa, Maku (Membrane), 5, 245 (1980).

31. P. M. Toporowski and J. E. Roovers, J. Polym. Sci., Polym. Chem. Ed., 14, 2233 (1976).

32. B. M. Seguela, M. S. Jacques, J. M. Renaud, and J. Prud'homme, Macromolecules, 13, 100 (1980).

33. A. Beamish, R. A. Goldberg, and D. J. Hourston, Polymer, 18, 49 (1977).

34. R. Seguela and J. Prud'homme, Macromolecules, 11, 1007 (1978).

35. B. Djermouni and H. J. Ache, Macromolecules, 13, 168 (1980). 